\title{
MENSURAÇÃO DA PRESSÃO ARTERIAL POR PALPAÇÃO DIGITAL DO PULSO PERIFÉRICO EM CÃES ANESTESIADOS
}

\author{
MEASUREMENT OF BLOOD PRESSURE BY DIGITAL PALPATION OF THE PERIPHERAL \\ PULSE IN ANESTHETIZED DOGS
}

\author{
T. A. RABELLO ${ }^{1}$, E. R. MONTEIRO ${ }^{1,2}$, D. CAMPAGNOL ${ }^{1}$, T. CHAMPION ${ }^{1,3}$, \\ T. F. BRESSAN ${ }^{1,4}$, K. COELHO ${ }^{1}$
}

\begin{abstract}
RESUMO
Com o presente estudo, buscou-se determinar a acurácia da aferição da pressão arterial (PA) pela palpação digital do pulso periférico (método indireto) em cães anestesiados. A determinação indireta da PA foi realizada com auxílio de manguito e esfigmomanômetro posicionados proximais ao carpo e o valor da PA foi considerado aquele registrado pelo esfigmomanômetro no momento em que a palpação do pulso retornava na artéria metacarpiana após a oclusão e subsequente desinsuflação gradual do manguito. A PA foi mensurada simultaneamente pelo método invasivo (direto). Os dados foram comparados pela análise de Bland Altman para verificar a concordância entre os métodos. Foram realizadas 131, 65 e 56 observações em 31 cães, respectivamente em normotensão, hipotensão e hipertensão. Durante a normotensão, hipotensão e hipertensão, os valores do viés para a pressão arterial sistólica (PAS) entre os métodos foram respectivamente $8,-2$ e $12 \mathrm{mmHg}$, demonstrando subestimação desta variável pelo método indireto na normotensão e hipertensão. Para a PAS, as diferenças entre os métodos que constituíram $<10$ e $<20 \mathrm{mmHg}$ foram respectivamente: hipotensão, 49 e 88\%; normotensão, 47 e 79\%; hipertensão, 25 e 52\%. Quando utilizado para a determinação da PAS em cães, o método de palpação digital do pulso periférico apresenta concordância inferior com o método invasivo em relação ao Doppler e similar em relação à PAS mensurada por diversos monitores oscilométricos relatados na literatura, mas não cumpre as exigências estabelecidas pelo CAMIV. O uso do método de palpação do pulso periférico para a monitoração da pressão arterial em cães durante a anestesia requer outros estudos para determinar com maior precisão a acurácia desse método.
\end{abstract}

PALAVRAS-CHAVE: Caninos. Monitoração cardíaca. Pressão arterial não invasiva. Pulso arterial.

\section{SUMMARY}

The present study aimed to determine the accuracy of measurement of blood pressure (BP) by digital palpation of peripheral pulse (indirect method) in anesthetized dogs. Indirect determination of BP was performed using a sphygmomanometer and a cuff placed proximal to carpus and BP was defined as the value recorded by sphygmomanometer at the time that digital palpation of the pulse in metacarpal artery returned after occlusion and subsequent gradual cuff deflation. Blood pressure was measured simultaneously by invasive (direct) method. Data were compared by Bland Altman analysis to verify the agreement between methods. A total of 131, 65 and 56 observations were performed in $31 \mathrm{dogs}$ during normotension, hypotension and hypertension, respectively. During hypotension, normotension and hypertension, values of bias for systolic arterial pressure (SAP) between methods were respectively 2, 8 and $12 \mathrm{mmHg}$, demonstrating underestimation of SAP by the indirect method during normotension and hypertension. Percentages of differences between methods for SAP that were $<10$ and <20 mmHg were: hypotension, 49 and 88\%; normotension, 47 and 79\%; hypertension, 25 and 52\%. For determination of SAP in dogs, indirect method reported here have lower agreement with the invasive method compared with Doppler and similar to many oscillometric monitors reported in the literature, but do not meet the requirements established by the ACVIM. Use of digital palpation of peripheral pulse for monitoring blood pressure during anesthesia in dogs requires further studies to more accurately determine the accuracy of this method.

KEY-WORDS: Arterial pulse. Canine. Cardiac monitoring. Noninvasive blood pressure.

\footnotetext{
${ }^{1}$ Universidade Vila Velha. Email.: thiagoarabello@ hotmail.com

${ }^{2}$ Universidade Federal do Rio Grande do Sul

${ }^{3}$ Universidade Federal da Fronteira Sul

${ }^{4}$ Faculdade de Zootecnia e Engenharia de Alimentos, Universidade de São Paulo
} 


\section{INTRODUÇÃO}

O método invasivo (direto) é considerado o padrão ouro para a mensuração da pressão arterial (PA), permitindo a amostragem continua de valores e a visualização das ondas de PA (WADDELL, 2000). Além disso, o acesso arterial estabelecido durante a mensuração direta da PA facilita a coleta de amostras de sangue arterial para avaliação hemogasométrica objetivando diagnóstico e tratamento de desequilíbrios ácido-base (WADDELL, 2000). Possíveis complicações relatadas após o uso do método direto foram hematoma no local de punção, de ocorrência frequente e trombose arterial, infecção e isquemia tecidual distal ao acesso arterial, estas últimas de ocorrência rara (WADDELL, 2000). A técnica consiste na introdução de cateter de calibre compatível em artéria periférica, ligado a extensor rígido previamente preenchido com solução heparinizada, podendo ser conectado a um esfigmomanômetro ou a um transdutor de pressão (WADDELL, 2000; HASKINS, 2007). As principais artérias utilizadas em cães e gatos são a femoral, a metatársica dorsal e a auricular (WADDELL, 2000).

Métodos não invasivos (indiretos) são alternativas para estimar os valores de PA. Como são estimativas da PA mensurada pelo método direto, todos os métodos indiretos apresentam algum erro e, para sua utilização na rotina clínica ou em pesquisas científicas, a concordância entre os métodos indiretos com 0 método direto deve ser determinada previamente segundo critérios estabelecidos pelo Colégio Americano de Medicina Interna Veterinária (CAMIV) (BROWN et al., 2007). Diversos métodos indiretos foram relatados na literatura tais como o Doppler (DYSON, 2007; GAROFALO et al., 2012), o método oscilométrico convencional (WERNICK et al., 2010; GAROFALO et al., 2012) e de alta definição (WERNICK et al., 2010) e a avaliação de ondas pletismográficas com uso de oxímetro de pulso (DYSON, 2007).

Todos os métodos indiretos anteriormente citados apresentam custo relativo de aquisição do equipamento. $\mathrm{O}$ método de palpação digital do pulso periférico é uma alternativa de baixo custo, sendo executado de forma similar ao Doppler diferindo deste último pelo fato de, ao invés de posicionar o transdutor do Doppler sobre uma artéria periférica, a detecção do pulso periférico é realizada pela palpação digital deste (HASKINS, 2007). O objetivo do presente estudo foi verificar a acurácia do método de palpação digital do pulso periférico da artéria metacarpiana para a mensuração da PA e estabelecer limites de concordância com a pressão arterial sistólica e média mensuradas pelo método invasivo, em condições de hipotensão, normotensão e hipertensão em cães anestesiados com isoflurano.

\section{MATERIAL E MÉTODOS}

Foram utilizados trinta e um cães (trinta fêmeas, um macho), com idade de 4,7 $\pm 4,0$ anos, com peso de
$11,2 \pm 5,7 \mathrm{~kg}$, procedentes da rotina cirúrgica do hospital veterinário da instituição.

Os animais foram anestesiados conforme a escolha do anestesista responsável, mas em todos os casos, a manutenção da anestesia foi realizada com isoflurano. Não foram utilizados fármacos vasoconstrictores. Durante a manutenção da anestesia, um cateter $22 \mathrm{G}$ foi introduzido por punção percutânea na artéria metatársica dorsal ou artéria femoral para permitir a mensuração da pressão arterial pelo método direto. O cateter arterial foi conectado a um sistema tubular rígido e transdutor de pressão ${ }^{5}$, previamente preenchido com solução heparinizada, para permitir a visualização dos valores de pressão arterial sistólica (PAS), média (PAM) e diastólica (PAD) na tela de um monitor multiparamétrico ${ }^{6}$. O transdutor de pressão foi zerado na altura do coração do animal e sua precisão testada antes de cada avaliação com o auxílio de uma coluna de mercúrio.

Simultaneamente ao registro dos valores de pressão arterial pelo método invasivo, a pressão arterial foi mensurada pelo método de palpação digital do pulso da artéria digital palmar (método indireto). Foi feita tricotomia na região palmar, imediatamente proximal ao coxim metacarpiano. No mesmo membro torácico, um manguito de pressão foi posicionado proximal ao carpo e conectado a um manômetro de mercúrio $^{7}$. A largura do manguito foi equivalente a aproximadamente $40 \%$ da circunferência do membro do animal. Para a mensuração da pressão arterial pelo método de palpação digital, o manguito de pressão foi insuflado com auxílio de uma pêra de borracha até que não fosse mais possível palpar o pulso da artéria digital palmar (aproximadamente $20 \mathrm{mmHg}$ acima do valor no qual ainda era possível palpar o pulso). Subsequentemente, o manguito era lentamente desinsuflado e a pressão pelo método de palpação digital foi considerada $\mathrm{o}$ valor observado no manômetro de mercúrio no momento exato em que o pulso retornava. Durante as mensurações da pressão arterial, o membro torácico do animal foi posicionado de forma que o manguito estivesse nivelado na mesma altura do coração do animal. Em cada momento, foram realizadas três mensurações consecutivas da pressão arterial pelo método de palpação digital, sendo essas consideradas quando a diferença entre elas foi menor do que $20 \mathrm{mmHg}$. A média das três mensurações foi utilizada para análise. Os valores de PAS, PAM e PAD pelo método invasivo foram simultaneamente registrados. Dois observadores foram responsáveis pelo registro dos valores de pressão. $\mathrm{O}$ observador responsável pela mensuração da pressão arterial pelo método de palpação digital possuía treinamento prévio, sendo responsável pela realização das mensurações em todas as ocasiões e desconhecia os valores de pressão mensurados pelo método invasivo, os quais foram

\footnotetext{
${ }^{5}$ Utah Medical Products, Athlone, County Westmeath, Republic of Ireland.

${ }^{6}$ LifeWindow 6000V, Digicare Biomedical

Technology, Boynton Beach, FL, USA.

${ }^{7}$ Oxigel, São Paulo, SP.
} 
registrados por um segundo observador. Os valores de pressão mensurados pelos dois métodos foram registrados em intervalos de cinco a dez minutos dependendo da extensão total do procedimento realizado.

Para análise, os dados foram agrupados baseado nos valores de PAS obtidos pelo método invasivo da seguinte forma: hipotensão, $\mathrm{PAS}<90 \mathrm{mmHg}$; normotensão, PAS 90 a 130 mmHg; e hipertensão, PAS $>130 \quad$ mmHg \& HELLEBREKERS 2008; GAROFALO et al., 2012). Os valores de pressão obtidos pelo método indireto foram comparados com os valores de PAS e PAM mensurados pelo método invasivo utilizando a análise de Bland-Altman para determinação dos erros médios entre os dois métodos (viés) e limites de concordância (viés $\pm 1,96$ vezes o desvio padrão do viés). Aprovado pelo Comitê de Ética da Instituição (protocolos: 195/2011 e 210/2011).

\section{RESULTADOS}

Em um animal, não foi possível efetuar nenhuma mensuração de pressão pelo método de palpação digital. Em dezessete ocasiões, observadas em seis animais, não foi possível efetuar mensurações indiretas simultaneamente com o método invasivo.

Foram realizadas um total de 252 mensurações simultâneas, sendo destas 131, 65 e 56 em normotensão, hipotensão e hipertensão, respectivamente. Os valores do viés foram menores e os limites de concordância mais estreitos quando o método indireto foi comparado aos valores de PAS do que aos valores de PAM obtidos pelo método invasivo (Tabela 1, Figura 1). As porcentagens das diferenças entre os dois métodos que se encontraram inferiores a $10 \mathrm{mmHg}$ e $20 \mathrm{mmHg}$, durante a normotensão, hipotensão e hipertensão encontram-se na Tabela 1.

Tabela 1 - Valores dos erros médios (viés[desvios padrão]), limites de concordância e porcentagens das diferenças que foram < 10 e $20 \mathrm{mmHg}$ observados na comparação dos valores de pressão mensurados pelo método indireto com os valores de pressão arterial sistólica (PAS) e média (PAM) mensurados pelo método direto em cães durante diferentes condições hemodinâmicas. Os dados de todas as variáveis referem-se a mmHg.

\begin{tabular}{|c|c|c|c|c|}
\hline & \multicolumn{4}{|c|}{ PAS invasiva $\mathrm{X}$ método indireto } \\
\hline & Viés (DP) & $\begin{array}{c}\text { Limites de } \\
\text { concordância }\end{array}$ & $<10 \mathrm{mmHg}(\%)$ & $<20 \mathrm{mmHg}(\%)$ \\
\hline Hipotensão & $-2(15)$ & -32 a 27 & 49,2 & 87,7 \\
\hline Normotensão & $8(14)$ & -18 a 35 & 47,3 & 78,6 \\
\hline \multirow[t]{3}{*}{ Hipertensão } & $12(25)$ & -38 a 61 & 25,0 & 51,8 \\
\hline & \multicolumn{4}{|c|}{ PAM invasiva $\mathrm{X}$ método indireto } \\
\hline & Viés (DP) & $\begin{array}{c}\text { Limites de } \\
\text { concordância }\end{array}$ & $<10 \mathrm{mmHg}(\%)$ & $<20 \mathrm{mmHg}(\%)$ \\
\hline Hipotensão & $-26(14)$ & -54 a 2 & 10,8 & 37,0 \\
\hline Normotensão & $-24(18)$ & -58 a 11 & 22,9 & 48,1 \\
\hline Hipertensão & $-18(24)$ & -65 a 29 & 28,6 & 57,2 \\
\hline
\end{tabular}

Quando os valores de pressão mensurados pelo método de palpação digital foram comparados aos valores de PAS mensurados pelo método invasivo, foi observado um número semelhante de observações subestimando e superestimando a PAS durante a hipotensão (viés -2 mmHg - Tabela 1, Figura 1). Das 65 observações realizadas durante a hipotensão, o método de palpação digital foi capaz de diagnosticar corretamente a existência de hipotensão em 51 observações (78\%). Durante normotensão e hipertensão, o método indireto subestimou a PAS mensurada pelo método invasivo na maioria das observações (viés positivo - Tabela 1, Figura 1). O método indireto foi capaz de diagnosticar corretamente a existência de normotensão em 89/131 (68\% das observações) e na hipertensão, o diagnóstico foi correto em 30/56 (54\% das observações).

Por outro lado, quando os valores de pressão obtidos pelo método indireto foram comparados aos valores de PAM invasiva, a grande maioria das observações superestimou os valores de PAM (valores de viés negativos - Tabela 1, Figura 1), sendo que durante a hipotensão, a PAM foi superestimada pelo método indireto em 64 das 65 observações (98\%), e durante a normotensão e hipertensão, a PAM foi superestimada em 123 de 131 (94\%) e em 43 de 56 $(77 \%)$ observações, respectivamente.

\section{DISCUSSAO}

De acordo com o CAMIV, um método não invasivo de mensuração da pressão arterial deve atingir as seguintes metas para ser considerado adequado para utilização clínica: viés $\pm 10 \mathrm{mmHg}$ com desvio padrão $\leq 15 \mathrm{mmHg}$; pelo menos $50 \%$ das observações com erro $\leq 10 \mathrm{mmHg}$ e pelo menos $80 \%$ das observações com erro $\leq 20 \mathrm{mmHg}$ (BROWN et al., 2007). No presente estudo, esses critérios ficaram bem próximos de serem atingidos nas condições de hipotensão e normotensão quando o método indireto foi utilizado como estimativa da PAS (Tabela 1). Vale ressaltar que nem todos os métodos indiretos atualmente utilizados para a mensuração da pressão arterial em cães preenchem os requisitos estabelecidos pelo CAMIV (BROWN et al., 2007). 

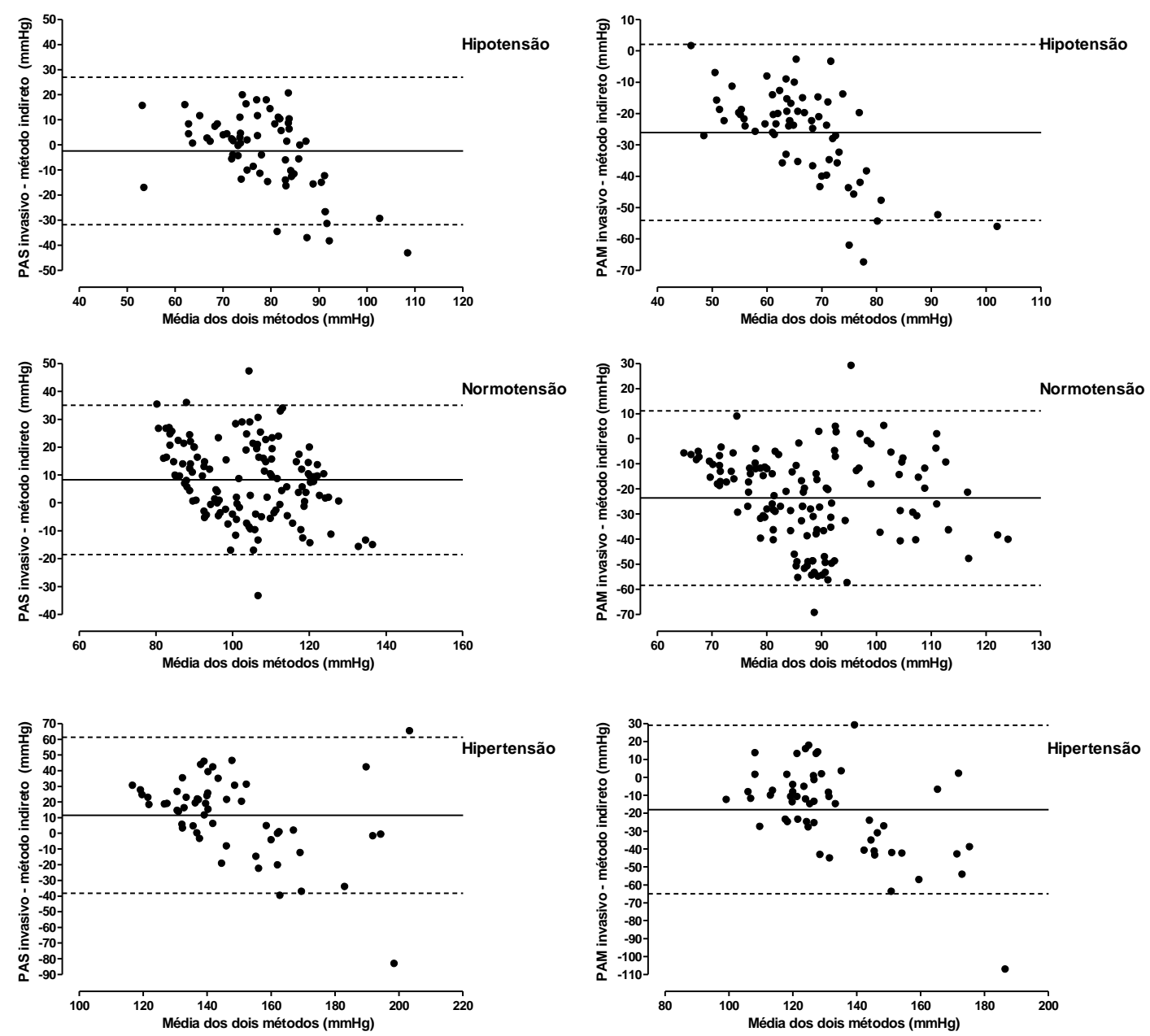

Figura 1 - Limites de concordância determinados pelo método de Bland-Altman em condições de normotensão, hipotensão e hipertensão. Cada ponto corresponde a 1 par de valores obtidos simultaneamente pelo método invasivo e o método de palpação digital do pulso periférico (indireto). A linha sólida preta representa o viés entre os valores de pressão arterial sistólica (PAS - coluna da esquerda) ou pressão arterial média (PAM - coluna da direita) obtidos pelo método invasivo e os valores obtidos pelo método indireto. As linhas tracejadas compreendem os limites de concordância. Os pontos acima do zero no eixo y caracterizam valores de pressão subestimados pelo método indireto enquanto os pontos abaixo desta linha caracterizam valores superestimados.

O Doppler é um método utilizado para mensurar, de forma não invasiva, os valores de PAS (HASKINS, 2007). O método utilizado no presente estudo é executado de forma similar ao Doppler, diferindo apenas pelo fato de que a detecção do pulso se faz por palpação digital e não por um transdutor. Foi relatado anteriormente que, quando o método de palpação do pulso periférico é empregado, durante a desinsuflação do manguito, a PAS corresponde à pressão no manômetro aneróide visualizada ao momento em que começam a ocorrer oscilações no ponteiro do manômetro; a pressão visualizada no manômetro no momento em que o pulso volta a ser palpável corresponde à PAD (HASKINS, 2007). Os resultados do presente estudo contradizem a literatura anterior uma vez que, levando-se em consideração a palpação do pulso periférico (e não oscilações no ponteiro do manômetro), foi demonstrada maior concordância entre os valores mensurados pelo método indireto com a PAS, sendo os valores de PAM superestimados pelo método indireto em todas as condições avaliadas (viés negativo na hipotensão, normotensão e hipertensão). Neste estudo, os valores mensurados pelo método indireto não foram comparados à PAD mensurada de forma invasiva porque obviamente esses valores seriam ainda mais superestimados do que a PAM.

Em um estudo anterior (GAROFALO et al., 2012), os autores relataram viés (limites de concordância) de 0,2 (-16 a 16 mmHg), -6 (-31 a 19 $\mathrm{mmHg}$ ) e -18 (-66 a $31 \mathrm{mmHg}$ ), respectivamente na hipotensão, normotensão e hipertensão, quando o transdutor do Doppler foi colocado sobre a artéria metatarsiana (na região plantar do membro pélvico) e o manguito posicionado proximal ao tarso. Esses resultados são similares aos deste estudo (Tabela 1) com exceção dos limites de concordância na hipotensão que foram mais amplos no presente estudo (-32 a $27 \mathrm{mmHg}$ ). Adicionalmente, em ambos os estudos, os valores de PAS foram subestimados pelo 
método não invasivo durante a normotensão e a hipertensão. Apesar das similaridades entre os estudos, durante condições de hipotensão e normotensão, as porcentagens de erros entre os métodos que ficaram $\leq 10$ e $20 \mathrm{mmHg}$ foram maiores com a utilização do Doppler do que com o método empregado nesse estudo e ficaram próximos dos critérios estabelecidos pelo CAMIV com o uso do Doppler (BROWN et al., 2007). Em outro estudo realizado em cães, porém com o Doppler posicionado no membro torácico, os resultados também demonstraram elevada concordância com o método invasivo (DYSON, 2007). Baseado nos resultados do presente estudo e dos estudos anteriores, é possível sugerir que o Doppler pode ser considerado um método mais confiável do que o método de palpação digital do pulso periférico para a monitoração da PAS em cães, especialmente durante condições de hipotensão e normotensão.

Outro método indireto frequentemente empregado para monitoração da pressão arterial em cães é o oscilométrico. Entretanto, resultados de estudos anteriores demonstraram uma grande variabilidade na acurácia desse método e sugerem que o método oscilométrico apresenta maior concordância com a PAM e PAD do que com a PAS mensurada pelo método invasivo (WERNICK et al., 2010; GAROFALO et al., 2012). No estudo realizado por GAROFALO et al. (2012), os valores do viés (limites de concordância) entre a PAS do método oscilométrico (com manguito posicionado proximal ao carpo) e a PAS invasiva, determinados durante a hipotensão, normotensão e hipertensão foram, respectivamente: $6(-9$ a 21$) \mathrm{mmHg}$, 15 (-37 a 7) mmHg e $-42 \quad(-84$ a -1$) \quad m m H g$. Adicionalmente, as diferenças entre os valores de PAS determinados pelos dois métodos que foram $\leq 10$ e 20 mmHg durante a hipotensão, normotensão e hipertensão foram, respectivamente: 67 e 100\%, 32 e $64 \%$, e 4 e $16 \%$. Sendo assim, somente durante a hipotensão, o monitor testado nesse estudo anterior atingiu as metas estabelecidas pelo CAMIV para a mensuração da PAS (BROWN et al., 2007). Em outro estudo realizado em cães, a acurácia de dois monitores oscilométricos diferentes, com manguito posicionado proximal ao carpo, foi avaliada em relação ao método invasivo (WERNICK et al., 2010). Neste estudo anterior, as mensurações de PAS efetuadas por um dos monitores oscilométricos apresentou elevada correlação com o método invasivo enquanto que o segundo monitor apresentou baixa correlação. Apesar da falta de acurácia na determinação da PAS por alguns monitores oscilométricos, especialmente na hipertensão, os valores de PAM mensurados por esses monitores demonstraram boa concordância com o método invasivo (WERNICK et al., 2010; GAROFALO et al., 2012). Assim como o Doppler, o método oscilométrico também parece apresentar maior acurácia e segurança na monitoração da pressão arterial em cães do que o método de palpação digital do pulso periférico, quando as decisões durante o uso desse monitor são tomadas baseadas nos valores de PAM e não de PAS.

No que se refere à avaliação qualitativa, os resultados do presente estudo foram similares aos resultados da avaliação quantitativa (concordância entre os métodos). O diagnóstico foi corretamente realizado em $78 \%$, 68\% e $54 \%$ das mensurações realizadas durante a hipotensão, normotensão e hipertensão, respectivamente. Baseado nesses resultados, em 22\% das mensurações em hipotensão e em $56 \%$ das mensurações em hipertensão, o tratamento para essas condições não seria adequadamente instituído. Esses resultados reforçam que a determinação da PAS pelo método de palpação digital deve ser realizada e interpretada diante dessas limitações.

Durante a hipotensão, quando os valores de pressão obtidos pelo método de palpação digital do pulso periférico foram comparados à PAM mensurada de forma invasiva, os valores foram superestimados pelo método indireto em 98,5\% das observações (64 de 65 observações - figura 1). Embora a concordância entre os métodos tenha sido fraca, levando-se em consideração a PAM, essa informação pode ter alguma utilidade quando esse método é empregado em cães. Esses resultados sugerem que, quando o valor mensurado pelo método de palpação digital for igual ou inferior a $60 \mathrm{mmHg} \mathrm{em}$ cães, o animal apresentará hipotensão (definida como PAM<60 $\mathrm{mmHg}$ ) (WADDELL, 2000; HASKINS, 2007) em quase $100 \%$ dos casos uma vez que o método superestima a PAM em 98,5\% das observações. Entretanto, essa abordagem não será útil na avaliação da resposta ao tratamento uma vez que não permite estimar com precisão os valores de PAM.

Foi demonstrado em estudo anterior que houve melhor concordância entre o Doppler e o método invasivo quando o transdutor foi colocado sobre a artéria metatarsiana e o manguito de pressão foi posicionado proximal ao tarso, do que distal ao tarso e também em relação ao uso do Doppler no membro torácico, com transdutor na artéria metacarpiana e o manguito posicionado proximal ao carpo (GAROFALO et al., 2012). Os valores do viés relatado nesse estudo anterior para o posicionamento do manguito proximal ao tarso, distal ao tarso e proximal ao carpo foram respectivamente: hipotensão, 0,2 , -6 e $10 \mathrm{mmHg}$; normotensão, -6, -13 e -3 mmHg; hipertensão, -18, -25 e -26 mmHg (GAROFALO et al., 2012). Resultados similares foram encontrados para o método oscilométrico, sendo o coeficiente de correlação entre os métodos indireto e direto de 0,69 para o membro pélvico e 0,53 para o membro torácico, independente da condição hemodinâmica do paciente (BODEY et al., 1994). É possível que, se no presente estudo, a determinação da PAS pela palpação digital do pulso periférico tivesse sido efetuada no membro pélvico, uma maior concordância com o método invasivo fosse evidenciada.

A avaliação da PAS por palpação digital do pulso periférico trata-se de um método que requer experiência pelo observador e apresenta um certo grau de subjetividade. Resultados diferentes podem ocorrer quando dois ou mais observadores utilizam este método. No presente estudo, um único observador, o qual foi treinado para a execução do método previamente ao início do estudo, foi responsável por realizar todas as mensurações. A utilização de dois observadores, mensurando a pressão arterial simultaneamente pelo método de palpação digital nos dois membros torácicos, 
poderia ter trazido informações adicionais a esse estudo tais como a determinação do coeficiente de variação inter-observador.

\section{CONCLUSÃO}

O método de palpação digital do pulso periférico é uma estimativa mais próxima da pressão arterial sistólica do que da pressão arterial média mensurada pelo método invasivo em cães anestesiados. Quando utilizado para a determinação da pressão arterial sistólica em animais desta espécie, esse método apresenta concordância inferior com o método invasivo em relação ao Doppler e similar em relação à pressão arterial sistólica mensurada por diversos monitores oscilométricos relatados na literatura, mas não se cumpre as exigências estabelecidas pela CAMIV. O uso do método de palpação digital do pulso periférico para a monitoração da pressão arterial em cães durante a anestesia requer outros estudos para determinar com maior precisão a acurácia desse método.

\section{REFERÊNCIAS}

BODEY, A. R.; YOUNG, L. E.; BARTRAM, D. H.; DIAMOND, M. J.; MICHELL, A. R. A comparison of direct and indirect (oscillometric) measurements of arterial blood pressure in anaesthetized dogs, using tail and limb cuffs. Research in Veterinary Science. v.57, p.265-269, 1994.

BROWN, S.; ATKINS, C.; BAGLEY, R.; CARR, A.; COWGILL, L.; DAVIDSON, M.; EGNER, B.; ELLIOTT, J.; HENIK, R.; LABATO, M.; LITTMAN, M.; POLZIN, D.; ROSS, L.; SNYDER, P.; STEPIEN, R. Guidelines for the identification, evaluation, and management of systemic hypertension in dogs and cats. Journal of Veterinary Internal Medicine. v.21, p.542-558, 2007.
DEFLANDRE, C. J. A.; HELLEBREKERS, L. J. Clinical evaluation of the surgivet V60046, a non invasive blood pressure monitor in anaesthetized dogs. Veterinary Anaesthesia and Analgesia. v.35, p.1321, 2008.

DYSON, D. H. Indirect measurement of blood pressure using a pulse oximeter in isoflurane anesthetized dogs. Journal of Veterinary Emergency and Critical Care. v.17, p.135-142, 2007.

GAROFALO, N. A.; TEIXEIRA NETO, F. J.; ALVAIDES, R. K.; OLIVEIRA, F. A.; PIGNATON, W.; PINHEIRO, R. T. Agreement between direct, oscillometric and Doppler ultrasound blood pressures using three different cuff positions in anesthetized dogs. Veterinary Anaesthesia and Analgesia. v.39, p.324-334, 2012.

HASKINS, S. C. Monitoring anesthetized patients. In: TRANQUILLI, W. J.; THURMON, J. C.; GRIMM, K. A. Lumb \& Jones' Veterinary Anesthesia and Analgesia. Ames: Blackwell Publishing, 2007. Cap.19, p.533-558.

WADDELL, L. S. Direct blood pressure monitoring. Clinical Techniques in Small Animal Practice. v.15, p.111-118, 2000.

WERNICK, M.; DOHERR, M.; HOWARD, J, FRANCEY, T. Evaluation of high-definition and conventional oscillometric blood pressure measurement in anaesthetised dogs using ACVIM guidelines. Journal of Small Animal Practice. v.51, p.318-324, 2010. 